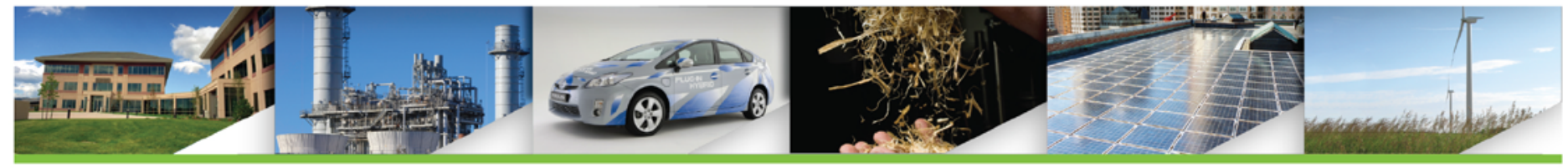

\title{
Managing the Electricity-Gas Interface: Current Environment and Emerging Solutions
}

Sean Ericson ${ }^{1}$, Jeffrey Logan ${ }^{1}$, and Daniel Kaffine ${ }^{1,2}$

${ }^{1}$ National Renewable Energy Laboratory

2 University of Colorado

Technical Report

NREL/TP-6A50-71750

July 2019

Contract No. DE-AC36-08G028308 


\section{Managing the Electricity-Gas Interface: Current Environment and Emerging Solutions}

Sean Ericson ${ }^{1}$, Jeffrey Logan ${ }^{1}$, and Daniel Kaffine $e^{1,2}$

${ }^{1}$ National Renewable Energy Laboratory

2 University of Colorado

The Joint Institute for Strategic Energy Analysis is operated by the Alliance for Sustainable Energy, LLC, on behalf of the U.S. Department of Energy's National Renewable Energy Laboratory, the University of Colorado-Boulder, the Colorado School of Mines, the Colorado State University, the Massachusetts Institute of Technology, and Stanford University.

JISEA $^{\circledR}$ and all JISEA-based marks are trademarks or registered trademarks of the Alliance for Sustainable Energy, LLC.

The Joint Institute for Strategic Energy Analysis 15013 Denver West Parkway Golden, CO 80401 303-275-3000 • www.jisea.org
Technical Report

NREL/TP-6A50-71750

July 2019

Contract No. DE-AC36-08GO28308 


\section{NOTICE}

This work was authored in part by the National Renewable Energy Laboratory, operated by Alliance for Sustainable Energy, LLC, for the U.S. Department of Energy (DOE) under Contract No. DE-AC36-08G028308. Funding provided by the Joint Institute for Strategic Energy Analysis. The views expressed herein do not necessarily represent the views of the DOE or the U.S. Government.

This report is available at no cost from the National Renewable Energy Laboratory (NREL) at www.nrel.gov/publications.

U.S. Department of Energy (DOE) reports produced after 1991 and a growing number of pre-1991 documents are available free via www.OSTI.gov.

Cover Photos: (left to right) NREL 04135, iStock 22779761, NREL 16933., NREL 15648, NREL 08466, NREL 21205

NREL prints on paper that contains recycled content. 


\section{List of Acronyms}

CCGT

EIA

FERC

HIFLD

ISO-NE

NERC

NGCC

NREL

PURPA

NREL combined cycle gas turbines

U.S. Energy Information Administration

Federal Energy Regulatory Commission

Homeland Infrastructure Foundation-Level Data

Independent Systems Operator of New England

North American Electric Reliability Corporation natural gas combined cycle

National Renewable Energy Laboratory

Public Utility Regulatory Policies Act

National Renewable Energy Laboratory 


\section{Executive Summary}

Natural gas generators are now the largest producers of electricity in the United States and the largest single end-use consumer of natural gas. Natural gas markets, which first developed to meet commercial and residential heating demands during winter months, are now entwined with electricity markets, which developed when natural gas provided a much lower fraction of total fuel supply for the electricity market. More coordination, both in daily operations and long-term planning, is required to ensure the continued supply of reliable electricity and natural gas. This report provides an overview of the evolution and current state of electricity and natural gas markets in the United States and summarizes the ways in which the two industries interact. The report describes areas warranting greater coordination between the natural gas and electricity industries. This report also discusses the current state of natural gas and electricity models and the need for integrated models that include dynamic simulations of pipeline operations, especially when large amounts of variable generation exists in the electrical system. It outlines current modeling work being done to better simulate operations and planning of both systems. 


\section{Table of Contents}

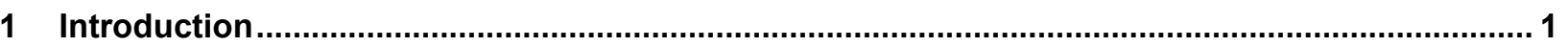

2 Electricity and Natural Gas Markets .................................................................................... 4

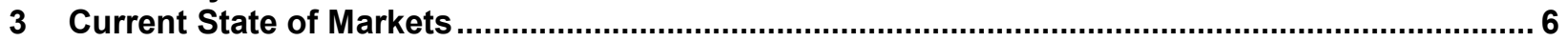

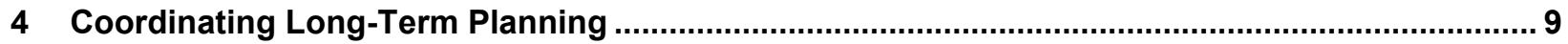

5 Coordinating Short-Run Gas Operations ......................................................................... 11

6 Current State of Gas-Electricity Modeling .......................................................................... 12

7 Conclusion and Policy Implications .................................................................................. 14

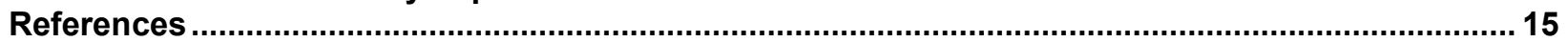




\section{List of Figures}

Figure 1. Total outages in the Northeast Power Coordinating Council by outage type versus temperature. 2 Figure 2. Annual electricity capacity additions in the United States by fuel type. Includes dates of major

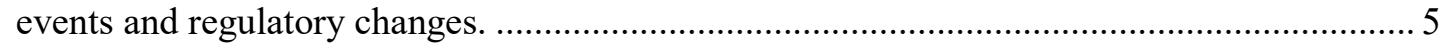

Figure 3. Map of U.S. interconnections, competitive wholesale electricity markets, and natural gas plants with capacity of at least $500 \mathrm{MW}$................................................................................... 7

Figure 4. Breakdown of U.S. natural gas consumption by end use and percentage of electricity generation

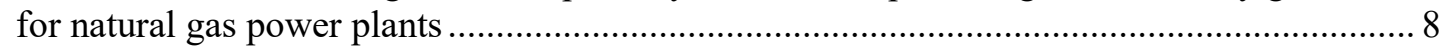




\section{Introduction}

Electricity produced from natural gas generation in the United States has increased dramatically in recent years, climbing from 13\% of total generation in 2001 to more than $35 \%$ in 2018 (EIA 2001-2018). Natural gas provides low-cost, dispatchable power and produces fewer emissions than other fossil fuels. The growth in natural gas generation has led to electricity and natural gas operations becoming increasingly interconnected. Natural gas-fired generators are now both the largest producer of electricity in the United States and the largest single end-use consumer of natural gas (EIA 2001-2018; EIA 2017). Efficient and reliable electrical grid and gas pipeline operations are dependent on effective coordination between the two markets.

The 2014 polar vortex made clear the need to better coordinate natural gas and electricity operations. In January 2014 , temperatures were $20-30^{\circ} \mathrm{F}$ below average for much of the Midwest, southern central region, and East Coast (NERC 2014). Frigid temperatures led to record electricity and natural gas demand (NERC 2014). At the same time, several power plants had their natural gas curtailed due to insufficient supply to meet both increased electricity and heating needs. As Figure 1 shows, fuel curtailment of natural gas to electric power plants during the polar vortex was a major contributor to electricity capacity reductions in the Northeast. While emergency procedures allowed the electrical grid to maintain reliability, the electrical system came dangerously close to not meeting demand.

Since the polar vortex, several steps have been taken to better coordinate natural gas and electricity markets and ensure reliability of both markets during the winter season. Some electricity system operators have instituted winter reliability programs and incentive mechanisms that reward availability during peak winter demand (Logan 2015). Transmission and pipeline infrastructure expansions are also underway in some areas, and energy efficiency and conservation measures have been enacted to alleviate constraints (Logan 2015); however, the growing interdependence between electricity and natural gas may require further steps be taken to ensure reliability of both systems going forward. 


\section{Northeast Power Coordinating Council}

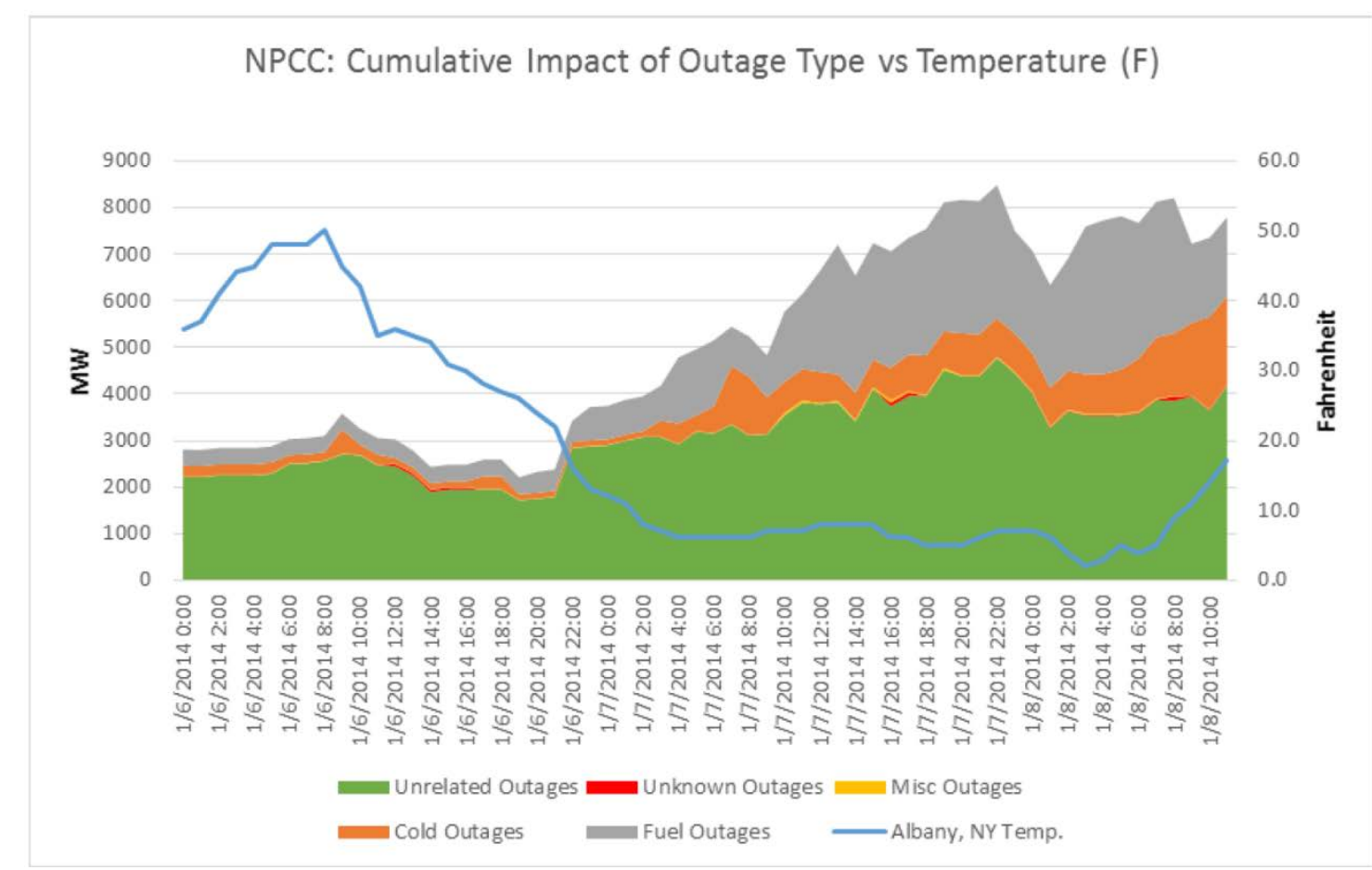

Figure 1. Total outages in the Northeast Power Coordinating Council by outage type versus temperature

Source: NERC (2014)

Gas-fired generation outages are increasing during winter months, especially in the northeastern United States (NERC 2017). Growth in natural gas demand from power plants leads to pipeline capacity constraints that bind more consistently. Electric reliability concerns stemming from natural gas supply issues are expected to become more acute in the coming years. A January 2018 study of different potential grid scenarios by the Independent Systems Operator of New England (ISO-NE) concludes that, "....the risk of future energy shortfalls is greater than the risk today. All but one of the 23 scenarios show that the regional power system could frequently experience some degree of system stress, requiring system operators to employ emergency procedures." While additional generation from wind and solar may reduce pipeline constraints, its variable nature may also lead to increased ramping of natural gas generation. This can place additional strain on natural gas infrastructure not originally designed to support fast changes in load (Eastern Interconnection States’ Planning Council 2014).

As demonstrated by the Aliso Canyon gas leak in Southern California, in addition to fuel supply risk, the electrical grid is exposed to single-point failures from natural gas infrastructure (NERC 2017). As electric compressors for natural gas transportation become more common, natural gas supply is also at risk from grid outages. In addition, the mutual dependence between electricity and natural gas operations increases the vulnerability of both to disruptions from physical and cyberattacks (Idaho National Laboratory 2016).

Greater coordination between grid operators and natural gas suppliers is required for the continued provision of reliable electricity and gas services. Coordination between electricity and 
natural gas operators can also lead to efficiency gains. Co-optimization of electricity and natural gas infrastructure can lead to lower costs compared to separate planning (Pambour et al. 2018). Greater communication and information sharing between the two markets can lead to more efficient generator dispatch and more efficient pipeline operations.

Integrated analysis of both electricity and gas operations is essential for facilitating coordination between electricity and natural gas operations. Sophisticated modeling tools that account for the dynamics and interactions between electrical grid and gas operations can allow operators to determine the most efficient and reliable way of providing electricity and natural gas.

This report provides an overview of the evolution and current state of electricity and natural gas markets and summarizes the ways in which the two industries interact. It discusses potential issues that warrant greater coordination between the two industries and outlines current modeling work being done to facilitate planning between the two. The first section gives a brief history of electricity and natural gas markets and summarizes the relevant workings of each. The second and third sections describe the need for long-term planning and short-run operational coordination. The fourth section discusses the current state of electricity and gas modeling, and discusses work being done to build integrated systems models to capture interactions between electricity and natural gas operations. The final section summarizes findings. 


\section{Electricity and Natural Gas Markets}

In many respects, electricity and natural gas markets share a parallel history. Both industries were structured as vertically integrated monopolies throughout most of the $20^{\text {th }}$ century, and both experienced significant market restructuring beginning in the 1980s. Importantly, changes in market structure have profoundly impacted the amount of natural gas used to generate electricity Flores-Espino et al. 2016).

Since the Public Utility Holding Company Act of 1935, the U.S. electrical system has been organized as a vertically integrated regulated monopoly. Because of the large transmission network required to connect producers to final consumers, a single firm can more efficiently provide transmission services than several competing firms. Under a vertically integrated monopoly system, to prevent inefficient duplication of capacity, a single utility is given the right to produce, transmit, and distribute electricity to customers within a designated service territory (Flores-Espino 2016). The utility's rate of return is then regulated to prevent it from exercising monopoly power to raise prices on customers. The Natural Gas Act of 1938 set up a very similar structure in the natural gas industry. The act established rules and rates for pipeline transportation and sales services (Oliver and Mason 2018). The Federal Power Commission oversaw regulation of prices for interstate natural gas production, transportation, and distribution (MacAvoy 2001).

While regulation and price controls under the regulated monopoly system protected customers from monopoly power, such controls were too slow to respond to changing costs of production. The regulations were also inadequate for taking account of regional price differences and the price controls often distorted firm incentives. In the natural gas industry, price controls led to underinvestment in production capacity, which in turn led to significant shortages of supply by the mid-1970s (MacAvoy 2001). In the winter of 1976-1977, the northern central region of the United States was "... short by as much as half of what industrial and commercial establishments on gas systems were calling for" (MacAvoy 2001). As natural gas markets were experiencing record shortages, a combination of rising oil prices and project cost overruns caused electricity prices to rise in the 1970s for the first time in eight decades (Blumsack et al. 2008).

Starting with the Natural Gas Policy Act of 1978 and ending with the Federal Energy Regulatory Commission (FERC) Order 636 in 1992, the natural gas industry went through a process known as restructuring. Price controls were removed from natural gas production, and owners of transmission pipelines divested their stakes in production and distribution capital (Oliver and Mason 2018). Beginning with the Public Utility Regulatory Policies Act (PURPA) of 1978 and taking form with FERC Orders 888 and 889 in 1996, the electricity markets began a similar restructuring process by allowing the price of electricity and some ancillary services to be determined by market forces.

Changes in market structure in the natural gas and electricity industries, in addition to the commercialization of efficient combined cycle gas turbines (CCGT), resulted in an unprecedented buildout of natural-gas fired generators. This buildout of natural gas generation capacity can be clearly seen in Figure 2, which displays a timeline of important market changes and net generation capacity additions. 
While the increase in capacity during the early 2000s was substantial, high gas prices resulted in a relatively low capacity factor for most gas plants. This changed with the prevalence of

hydraulic fracturing, which significantly reduced natural gas prices. Henry Hub ${ }^{1}$ spot prices fell by $64 \%$ from 2008-2018 (EIA 2018). Lower gas prices resulted in gas-fired power plants being dispatched more frequently, leading to higher average capacity factors (Fell and Kaffine 2018). The combined effects of more gas capacity and more average generation from gas plants was a doubling of the share of natural gas used in power plants - from $20 \%$ in 1997 to nearly $40 \%$ in 2017 (EIA 2017).

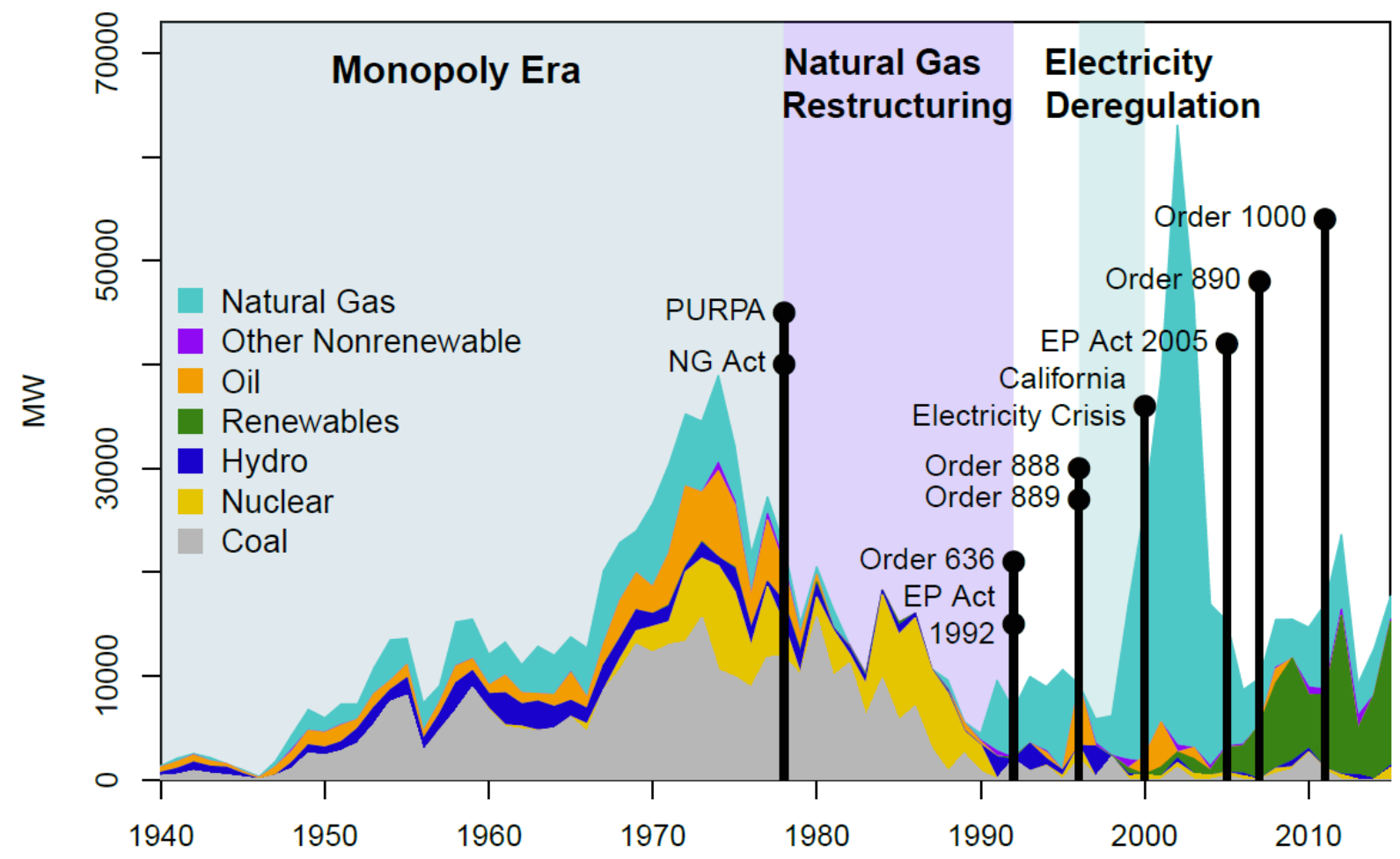

Figure 2. Annual electricity capacity additions in the United States by fuel type. ${ }^{2}$ Includes dates of major events and regulatory changes.

Source: EIA

\footnotetext{
${ }^{1}$ Henry Hub, located in in Louisiana, is the official delivery location for futures contracts on the New York Mercantile Exchange and is often used as a benchmark for natural gas prices in North America.

${ }^{2}$ Figure displays capacity additions, meaning yearly net capacity additions are lower than displayed due to plant retirements.
} 


\section{Current State of Markets}

Today, natural gas markets have a clear separation between production, transportation, and distribution. While gas prices are determined by market forces, transmission and distribution prices are still regulated. Transmission rates vary depending on priority of service, with the more expensive firm contracts having priority over less-expensive interruptible contracts. During periods of high demand - when demand exceeds pipeline capacity_interruptible contracts are the first to be curtailed (FERC 1999). Firm contracts consist of both a variable charge for the gas consumed and a reservation charge, which is independent of the amount of gas used (FERC 1999). Interruptible contracts, on the other hand, only depend on the amount of gas used (FERC 1999). As utilization of gas-fired generators is uncertain, and because firm contracts are more expensive, natural gas generators primarily buy interruptible contracts. This means natural gas plants are the first to be curtailed when gas pipeline capacity becomes constrained.

While the United States has a restructured natural gas market, ${ }^{3}$ the market structure for electricity is more complicated. The process of deregulating wholesale electricity markets abruptly halted after a flawed market design led to the California electricity crisis of 2000 (Borenstein et al. 2002). While restructuring has continued since 2000 , roughly $40 \%$ of customers are still served by vertically integrated monopolies. Figure 3 displays the seven restructured markets in the United States, along with the areas (in white) still under a vertically integrated structure. Figure 3 also displays the three synchronized grid interconnections and all natural gas plants with a capacity of $500 \mathrm{MW}$ or more.

\footnotetext{
${ }^{3}$ The price of wholesale natural gas in the United States is determined by market forces; however, the retail price of natural gas is still often set by regulation.
} 


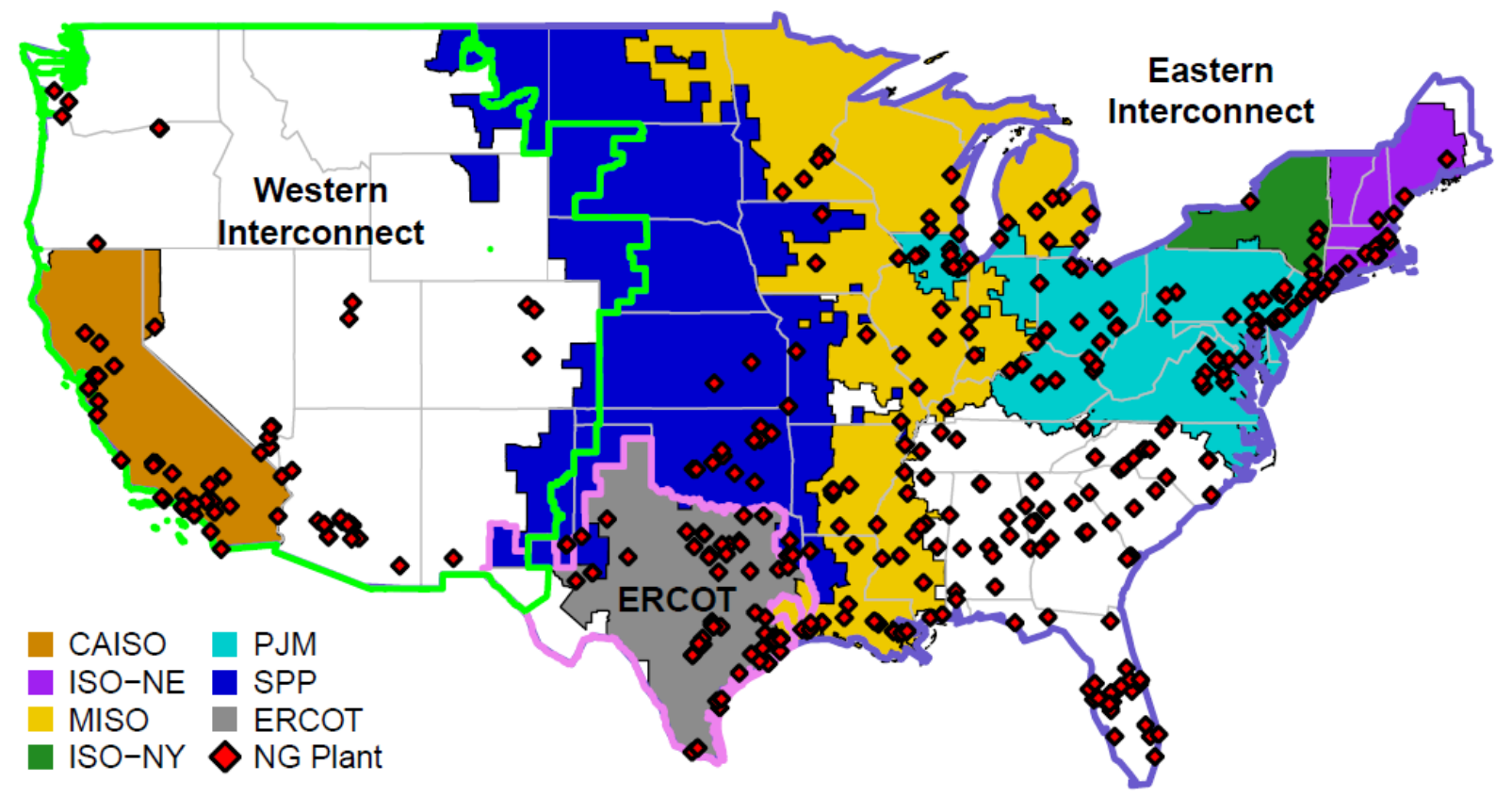

Figure 3. Map of U.S. interconnections, competitive wholesale electricity markets, and natural gas plants with capacity of at least $500 \mathrm{MW}$

Source: HIFLD (2017)

In restructured electricity markets, generator dispatch and the price of electricity are determined by the process of security-constrained economic dispatch (Flores-Espino et al. 2016). The process determines the lowest-cost means of meeting both load and system reliability constraints. Reliability constraints are mandated by the North American Electric Reliability Corporation (NERC). Given reliability constraints are met, generators are dispatched each hour by cost of production, with lower-cost generators being dispatched first. Most plants bid into a day-ahead market, where generators are dispatched hourly, so expected supply meets expected load. A realtime market, generally dispatched at 5- or 15-minute intervals, accounts for differences due to unexpected demand, generator outages, and forecast errors from wind and solar.

Recent low natural gas prices have led to natural gas combined cycle (NGCC) plants displacing coal plants on the dispatch order in many regions of North America (Logan et al. 2015). The average capacity factor for NGCC plants increased from 36\% in 2005 to more than 55\% in 2015 (Logan et al. 2017). Increases on the extensive margin, from a buildout of natural gas capacity, and on the intensive margin, from higher utilization rates due to low gas prices, have resulted in power plants becoming the primary customer for natural gas, as well as natural gas generating more electricity than any other fuel. Figure 4 displays the breakdown of natural gas consumption by end use in the United States over time, and shows the percentage of total electricity generation from natural gas power plants. Figure 4 also shows the increasing consumption of natural gas from power plants due to the rapid increase in natural gas generation over the last two decades. 


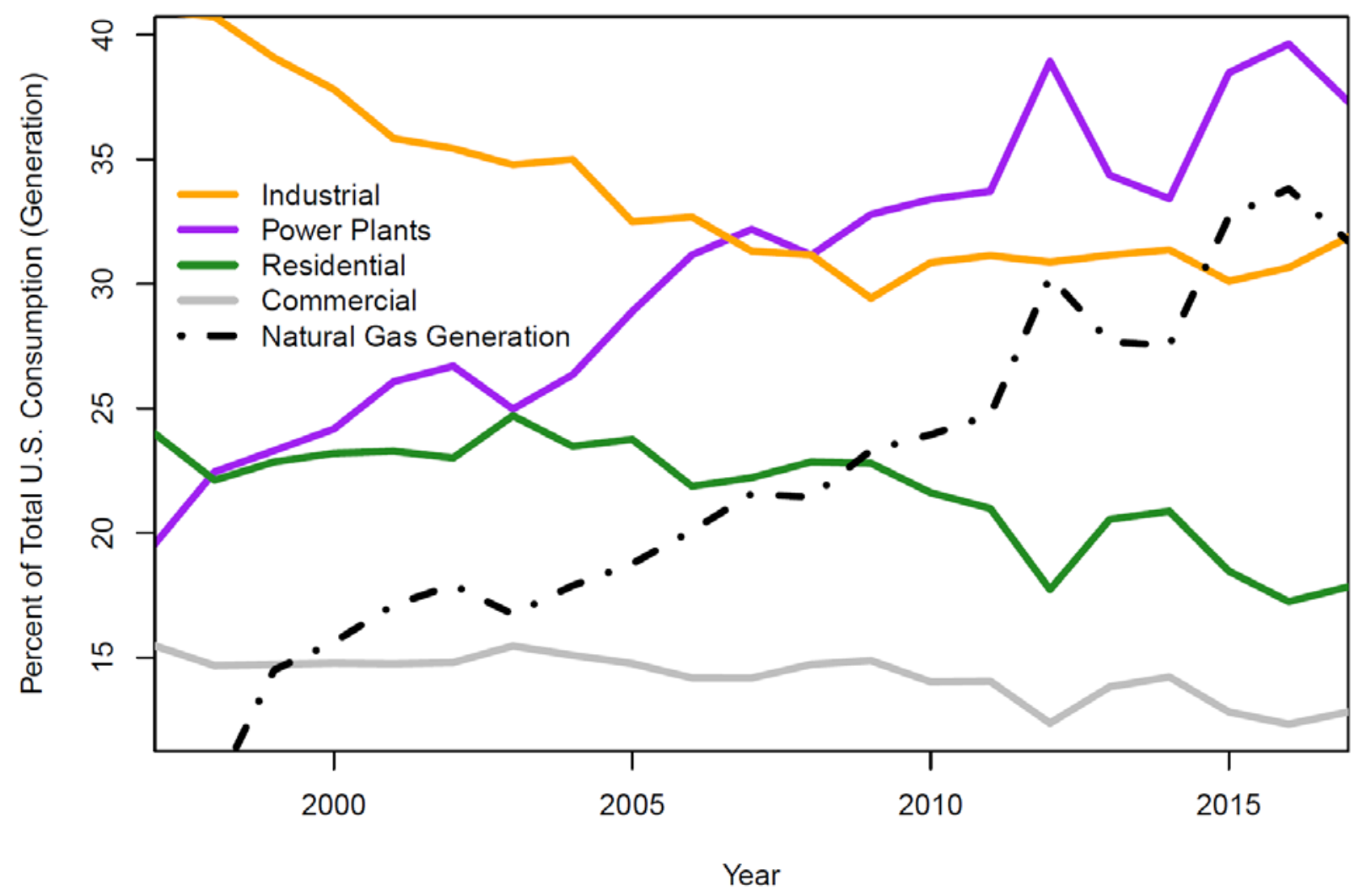

Figure 4. Breakdown of U.S. natural gas consumption by end use and percentage of electricity generation for natural gas power plants

Source: Consumption data from EIA (2017) and generation data from EIA

Natural gas, which, until recently, was used primarily for industrial and manufacturing processes, as well home heating and cooking, is now deeply entwined with the electrical system. Most forecasts show natural gas providing an even higher percentage of total generation in the coming years (ISO-NE 2018). Continued growth in variable generation from renewable energy will also likely require larger and more frequent ramping of natural gas plants to stabilize swings in net load. Additional regulations and changes to market operations may be required to maintain gas pipeline and electric system reliability with higher penetrations of natural gas and variable renewable generation. 


\section{Coordinating Long-Term Planning}

Coordinated long-term planning of both electricity and natural gas infrastructure is essential for maintaining reliability and efficiency in both industries. Optimizing the joint gas-electric infrastructure can ensure sufficient gas capacity for generation units, increase system flexibility, and reduce gas curtailments (Zhang et al. 2015; Saldarriaga et al. 2015; Eastern Interconnection States' Planning Council 2014). Currently, however, the reliability-driven planning of electricity projects is mismatched with the market-driven planning in the natural gas industry (Eastern Interconnection States' Planning Council 2014). Infrastructure in each industry is built with little consideration for the other.

The lack of coordination in long-term planning is partly a result of regulatory structures. A demonstration of "public convenience and necessity" to FERC is required before a new natural gas pipeline can be built (Oliver and Mason 2018). This generally requires the pipeline developer demonstrate that enough customers will buy long-term firm capacity (Oliver and Mason 2018). Because natural gas generators for electricity tend to purchase interruptible contracts due to insufficient incentive to procure firm capacity, pipelines are not built to accommodate natural gas generators (ISO-NE 2018).

Natural gas pipeline capacity constraints due to competing end uses for natural gas already lead to curtailments for gas-fired generation during winter months, when natural gas is needed for heating; increasing levels of gas-fired generation will only increase constraints in the coming years. Added variable generation capacity from wind and solar may reduce gas generation requirements, thereby alleviating some of these constraints, but the additional variable generation may also further complicate pipeline operations (Hibbard and Schatzki 2012; Nasr and Connor 2014). Natural-gas fired generators are ramped, among other reasons, to accommodate variable generation from wind and solar; however, gas pipelines are designed primarily to supply relatively steady residential heating needs and may be strained to accommodate such large rapid changes in demand. Further planning may be required to ensure the gas infrastructure can support both increasing levels of gas-fired generation and growing levels of variable generation.

Changes in market regulations to support construction of gas pipelines and gas storage to supply natural gas generators may be beneficial. Changes in market rules, such as pay for performance rules, may incentivize generators to secure firm contracts and lead to pipeline capacity expansion. Alternative means of ensuring reliability, such as supporting dual fuel use, ${ }^{4}$ increased gas storage, or improved demand response may be cheaper than natural gas pipeline expansion (Hibbard and Schatzki 2012).

Further research can consider how to jointly optimize the buildout of future electrical system and natural gas capacity. This would allow planners to determine areas that face the most risk from supply shortages and determine the most cost-effective solution.

Along with potential reliability issues from unintentional outages, the increasing interdependence between electricity and natural gas raises the threat of intentional disruptions, (i.e., from cyberattacks). The number and sophistication of cyberattacks on U.S. utilities has increased

\footnotetext{
${ }^{4}$ Dual fuel use is when a natural gas generator also can use fuel oil, which may be stored on-site.
} 
markedly in the last few years (Idaho National Laboratory 2016). Integration of electric and natural gas operations increases the number of attack vectors - the number of ways a malicious agent can gain access to disrupt operations - that both networks can be susceptible to. The electrical system is now at risk from malicious disruption of natural gas supply, and gas transmission is similarly at risk from grid disruptions. Further coordination between the electricity and oil and gas industries may be beneficial for determining how best to fortify against potential attacks (Eastern Interconnection States' Planning Council 2014). 


\section{Coordinating Short-Run Gas Operations}

Better coordination between electricity and natural gas operations can enhance efficiency and improve reliability. Information sharing between electricity and natural gas operators can reduce intermarket inefficiencies and support co-optimizing operations. Reliability can also be improved by taking steps to reduce the effect of disruptions in one market on the operations of the other.

Electricity and gas trading days are not synchronized, meaning generators must bid into electricity markets without knowing whether they will have sufficient gas supplies and bid into gas markets without knowing if they will be dispatched (FERC 2015). The electricity trading day begins at midnight to accommodate the morning ramp, while the natural gas trading day begins at 9 a.m. to allow workers the benefit of natural light for conducting operations (FERC 2015). Due to these operational differences, gas and electricity trading days are likely to stay separate for the foreseeable future. Improved coordination between the two markets can help mitigate market inefficiencies caused by the differences in trading days.

A single point failure in natural gas transportation can affect multiple generators, severely diminishing grid stability and potentially increasing electricity prices. An analysis by the Independent System Operator of New England concluded that a major compressor outage or an outage at a liquified natural gas terminal "would result in frequent energy shortages that would require frequent and long periods of rolling blackouts" (ISO-NE 2018). At the same time, some compressor stations use electric generators, implying a blackout can threaten the ability to distribute natural gas (Eastern Interconnection States' Planning Council 2014). Coordination between the two industries is therefore essential for maintaining grid reliability and reliable pipeline operations.

Separate optimization of electricity and natural gas operations can be less efficient than cooptimization between the two markets (Pambour et al. 2018). Co-optimization is largely constrained at the operations level by a lack of shared information and insufficient modeling capabilities. Generators are currently dispatched without consideration of the availability of natural gas (Eastern Interconnection States' Planning Council 2014). Knowledge of natural gas supply constraints allow grid operators to dispatch generators more efficiently, and better knowledge of generator operations help pipeline operators more efficiently schedule gas flows. Apart from potential privacy issues and difficulties in data sharing between industries, coordination between electricity and natural gas is constrained by limited modeling capabilities. Current electric dispatch models generally do not have the capability of considering natural gas network constraints. 


\section{Current State of Gas-Electricity Modeling}

Electricity and natural gas market models are constrained optimization problems, meaning the model finds the lowest-cost way to meet demand while also meeting operation constraints, such as generator, transmission, and pipeline capacity constraints. Electricity-gas interactions are captured by their effect on prices, which affect the model's cost equations, and their effect on operational constraints.

Gas and power systems have traditionally been modeled independently, with the implicit assumption that sufficient natural gas capacity exists to satisfy generator demand (Pambour et al. 2018). In an independent model, gas prices enter as an exogenous parameter and all operation constraints from gas-electric interactions are assumed to be non-binding. Many tools are available for modeling electricity or gas systems in isolation; however, the growing interdependence between gas and power systems makes accurate modeling of the electric-natural gas interface essential for facilitating coordination between electricity and gas operations. An integrated power and gas model accounts for the interactions of prices and physical constraints on each system. Few models can conduct an integrated analysis of electricity and gas systems, and even fewer can conduct an analysis at the scale required for grid operators (Erdener et al. 2014). A small but growing body of research explicitly accounts for electricity and natural gas interactions (Pambour et al. 2017; Zlotnik et al. 2017; Jiang et al. 2018).

Price dynamics between electricity and gas markets are important for capacity expansion models, which have time frames on the order of years to decades. A few analyses model the effects of natural gas supply on the utilization of gas generators (Cole et al. 2016). For integrated models of day-to-day grid operations, on the other hand, gas prices can be considered exogenous, and operation constraints are of primary concern.

Complicated system dynamics make modeling an integrated electric-gas system difficult (Pambour et al. 2018). Correctly modeling the dynamic nature of natural gas flows requires the model to solve partial differential equations, which can be computationally demanding. Most interconnected models therefore simplify the problem by assuming a steady state, where the flow of natural gas into a pipeline equals the flow out at any given time. Li et al. 2016, MartinezMares and Fuerte-Esquival 2011, and Alabdulwahab et al. 2015 are examples of the state of the art of steady-state analysis of interconnected power and gas operations.

While steady-state analysis can be valuable, it is insufficient for determining efficient operation and ensuring reliability. Using a static model of natural gas pipeline operations can lead to significant modeling errors (Correa-Posada and Sanchez-Martin 2015). To properly model interconnected electricity and gas operations, natural gas dynamics must be accounted for. Natural gas flows through pipelines relatively slowly, around 20-30 miles per hour. Points of supply and demand are often significant distances apart, meaning that meaningful lags occur between changes in demand and changes in supply. Therefore, large changes in gas demand can lead to abrupt changes in pressure, which lead to operational constraints. Furthermore, due to line packing - storing natural gas in the pipeline - gas supply can temporarily exceed the steadystate flow rate of a line. Demand and supply are constantly changing, and changes in linepack balances these fluctuations. Thus, a steady-state model cannot be used for operational analysis where an adequate reflection of the changes in pressure and linepack are necessary to account for 
physical constraints of the system. Generator ramping and grid contingencies lead to further complications that require dynamic modeling of natural gas (Pambour et al. 2018).

Some work has been done to develop an integrated framework that accounts for both grid constraints and dynamic natural gas operations (Pambour et al. 2018; Pambour et al. 2017; Correa-Posada and Sanchez-Martin 2015; Liu et al. 2011; Pambour et al. 2017). This research is beginning to answer the wide array of questions related to coordinating gas and electric operations (Pambour et al. 2018).

Ongoing research is further developing state-of-the-art methods and tools for co-simulation of natural gas and power system networks. Integrated simulation systems allow for better coordinating of electricity and natural gas markets, which can benefit both markets. Integrated simulations capture system interactions which separate platforms cannot model. Simulating the dynamic aspects of gas operations further captures the important effects of linepack and time delays in the gas network.

Electricity and gas networks are becoming more interconnected each year, and more is being asked of gas operations to accommodate growing penetration of variable generation. Integrated modeling is useful for providing a greater understanding of how electricity and gas markets can best coordinate operations, and what steps may need to be taken to ensure reliable operation in the long-term. Continued research and efforts to further the capabilities of integrated gaselectricity modeling, as well as further coordination between electricity and gas operations, is warranted. 


\section{Conclusion and Policy Implications}

Electricity and natural gas markets are interconnected and will grow increasingly interdependent. Natural gas plants generate more electricity in the United States than any other fuel source, and natural gas plants are the primary consumer of gas. Regulations and market structure for the natural gas and electricity industries are still evolving to account for the increased integration of the two markets. As the share of electricity from natural gas grows, efficiency and reliability issues are exacerbated.

More coordination, both with daily operations and long-term planning, is required to ensure the continued supply of reliable electricity and natural gas. Communication between electricity and gas operators is crucial to ensure reliable daily operations. Regulations and prices that allow and incentivize joint optimization of investments are essential for efficient long-term capacity expansion.

To facilitate coordination between markets, modeling tools that provide integrated analysis of optimal generator and gas dispatch are required; however, most integrated models use steadystate analysis, which is insufficient to capture the dynamic nature of natural gas operations. Current efforts are combining dynamic models of gas networks with electricity models into a single integrated framework. Continued research will further our understanding of how to gain the benefits of natural gas generation without risking electricity and gas reliability in the process. 


\section{References}

Alabdulwahab, Ahmed, Abdullah Abursorrah, Xiaping Zhang, and Mohammed Shahidehpour. "Coordination of Interdependent Natural Gas and Electricity Infrastructures for Firming the Variability of Wind Energy in Stochastic Day-Ahead Scheduling." IEEE Transactions on Sustainable Energy 6, no. 2 (April 2015): 606-615. https://ieeexplore.ieee.org/document/7060725.

Blumsack, Seth, Lester Lave, and Jay Apt. Electricity Prices and Costs Under Regulation and Restructuring. Carnegie Mellon Electricity Industry Center Working Paper. CEIC-0803. 2008. https://www.cmu.edu/ceic/assets/docs/publications/working-papers/ceic-0803.pdf.

Borenstein, Severin, James Bushnell, and Frank Wolak. "Measuring Market Inefficiencies in California's Restructured Wholesale Electricity Market." American Economic Review 92, no. 5 (February 2002): 1376-1405.

Cole, Wesley, Kenneth Medlock III, and Aditya Jani. "A view to the future of natural gas and electricity: An integrated modeling approach." Energy Economics 60 (November 2016): 486-496. https://doi.org/10.1016/j.eneco.2016.03.005.

Correa-Posada, Carlos, and Pedro Sanchez-Martin. "Integrated Power and Natural Gas Model for Energy Adequacy in Short-Term Operation." IEEE Transactions on Power Systems 30, no. 6 (November 2015): 3347-3355.

https://ieeexplore.ieee.org/stamp/stamp.jsp?arnumber $=6977991$.

Eastern Interconnection States' Planning Council, "Long-term Electric and Natural Gas Infrastructure Requirements." Illinois Institute of Technology. 2014. https://pubs.naruc.org/pub/536DCA2E-2354-D714-5112-CBD58ED9B81A

EIA (U.S. Energy Information Administration). "Henry Hub Natural Gas Spot Price." Natural Gas. Last modified July 10, 2019. https://www.eia.gov/dnav/ng/hist/rngwhhdD.htm

EIA. "Natural Gas Consumption by End Use.” Natural Gas. Last modified June 28, 2019. https://www.eia.gov/dnav/ng/ng_cons_sum_dcu_nus_a.htm.

Erdener, Burcin, Kwabena Pambour, Ricardo Bolado Lavin, and Berna Dengiz. "An Integrated Simulation Model for Analysing Electricity and Gas Systems." International Journal of Electrical Power \& Energy Systems 61 (October 2014): 410-420. https://doi.org/10.1016/j.ijepes.2014.03.052.

Fell, Harrison, and Daniel Kaffine. "The Fall of Coal: Joint Impacts of Fuel Prices and Renewables on Generation and Emissions." American Economic Journal: Economic Policy 10, no. 2 (May 2018): 90-116. https://www.aeaweb.org/articles?id=10.1257/pol.20150321. 
FERC. "Coordination of the Scheduling Processes of Interstate Natural Gas Pipelines and Public Utilities." Federal Register. Washington, D.C.: FERC. A Notice by the Federal Regulatory Commission on August 6, 2015. https://www.federalregister.gov/documents/2015/08/06/2015-19292/coordination-of-thescheduling-processes-of-interstate-natural-gas-pipelines-and-public-utilities.

FERC. Cost-of-Service Rates Manual. Washington, D.C.: FERC. June 1999.

Flores-Espino, Francisco, Tian Tian, Ilya Chernyakhovskiy, Megan Mercer, and Mackay Miller. Competitive Electricity Market Regulation in the United States: A Primer. Golden, CO: NREL. NREL/TP-6A20-67106. December 2016. https://www.nrel.gov/docs/fy17osti/67106.pdf.

Hibbard, Paul, and Todd Schatzki. "The Interdependence of Electricity and Natural Gas: Current Factors and Future Prospects." The Electricity Journal 25, no. 4 (May 2012): 6-17. https://doi.org/10.1016/j.tej.2012.04.012.

HIFLD (Homeland Infrastructure Foundation-Level Data). "HIFLD Open Data.” 2017. Accessed April 7, 2018. https://hifld-geoplatform.opendata.arcgis.com/.

Idaho National Laboratory. Cyber Threat and Vulnerability Analysis of the U.S. Electric Sector. Idaho Falls, ID: Idaho National Laboratory. August 2016. https://www.energy.gov/sites/prod/files/2017/01/f34/Cyber\%20Threat $\% 20$ and $\% 20$ Vulnerab ility $\% 20$ Analysis $\% 20$ of $\% 20$ the $\% 20$ U.S. $\% 20$ Electric $\% 20$ Sector.pdf.

ISO-NE. Operational Fuel-Security Analysis. Holyoke, MA: ISO-NE. January 2018. https://www.iso-ne.com/static-assets/documents/2018/01/20180117 operational fuelsecurity analysis.pdf.

Jiang, Yibo, Jian Xu, Yuanzhang Sun, Congying Wei, Jing Wang, Siyang Liao, Deping Ke, et al. "Coordinated operation of gas-electricity integrated distribution systems with multiCCHP and distributed renewable energy sources." Applied Energy 211 (February 2018): 237-248. https://doi.org/10.1016/j.apenergy.2017.10.128.

Li, Guoqing, Rufeng Zhang, Houhe Chen, Tao Jiang, Hongjie Jia, Yunfei Mu, and Xiaolong Jin. "Security-constrained economic dispatch for integrated natural gas and electricity systems." Energy Procedia 88 (June 2016): 330-335.

https://doi.org/10.1016/j.egypro.2016.06.140.

Liu, Cong, Mohammad Shahidehpour, and Jianhui Wang. "Coordinated scheduling of electricity and natural gas infrastructures with a transient model for natural gas flow." Chaos: An Interdisciplinary Journal of Nonlinear Science 21 (June 2011): 025102. https://doi.org/10.1063/1.3600761.

Logan, Jeffrey, Cara Marcy, James McCall, Francisco Flores-Espino, Aaron Bloom, Jorn Aabakken, Wesley Cole, et al. Electricity Generation Baseline Report. Golden, CO: NREL. NREL/TP-6A20-67645. January 2017. https://www.nrel.gov/docs/fy17osti/67645.pdf. 
Logan, Jeffrey, Kenneth Medlock, and William Boyd. A Review of Sector and Regional Trends in U.S. Electricity Markets: Focus on Natural Gas. Golden, CO: JISEA at NREL. NREL/TP-6A50-64652. October 2015. https:/www.nrel.gov/docs/fy16osti/64652.pdf.

MacAvoy, Paul. The Natural Gas Market: 60 Years of Regulation and Deregulation. New Haven, CT: Yale University, 2001.

Martinez-Mares, Alberto, and Claudio Fuerte-Esquivel. "Integrated energy flow analysis in natural gas and electricity coupled systems." in 2011 North American Power Symposium, Boston, MA. August 4-6, 2011. https://ieeexplore.ieee.org/abstract/document/6024880.

Nasr, Ghasem, and N. E. Connor. "Transmission and Distribution Systems and Design," in Natural Gas Engineering and Safety Challenges: Downstream Process, Analysis, Utilization and Safety. 17-43. New York City: Springer International Publishing, 2014.

NERC (North American Electric Reliability Corporation). Polar Vortex Review. Atlanta, GA: NERC. September 2014.

https://www.nerc.com/pa/rrm/January\%202014\%20Polar\%20Vortex\%20Review/Polar_Vor tex Review 29 Sept 2014 Final.pdf.

NERC. Special Reliability Assessment: Potential Bulk Power System Impacts Due to Severe Disruptions on the Natural Gas System. Atlanta, GA: NERC. November 2017.

https://www.nerc.com/pa/RAPA/ra/Reliability\%20Assessments\%20DL/NERC_SPOD_111 42017 Final.pdf.

Oliver, Matthew, and Charles Mason. "Natural Gas Pipeline Regulation in the United States: Past, Present, and Future." Foundations and Trends in Microeconomics 11, no. 4 (May 2018): 227-288. https://www.nowpublishers.com/article/Details/MIC-070.

Pambour, Kwabena, Burcin Erdener, Ricardo Bolado-Lavin, and Gerard Dijkema. "Development of a Simulation Framework for Analyzing Security of Supply in Integrated Gas and Electric Power Systems." Applied Sciences 7, no. 1 (January 2017): 47. https://pdfs.semanticscholar.org/973c/cd4b07b5b93459037d3bc84f6be6e12eae6d.pdf.

Pambour, Kwabena, Burcin Erdener, Ricardo Bolado-Lavin, and Gerard Dijkema. "SAInt A novel quasi-dynamic model for assessing security of supply in coupled gas and electricity transmission networks." Applied Energy 203 (October 2017): 829-857.

https://doi.org/10.1016/j.apenergy.2017.05.142.

Pambour, Kwabena, Rostand Sopgwi, Bri-Mathias Hodge, and Carlo Brancucci. "The Value of Day-Ahead Coordination of Power and Natural Gas Network Operations." Energies 11, no. 7 (June 2018). DOI: 10.3390/en11071628.

Saldarriaga, Carlos, Ricardo Hincapie, and Harold Salazar. "A Holistic Approach for Planning Natural Gas and Electricity Distribution Networks." IEEE Transactions on Power Systems 28, no. 4 (November 2013): 4052-4063. 
Zhang, Xiaping, Mohammad Shahidehpour, Ahmed Alabdulwahab, and Abdullah Abusorrah. "Optimal Expsansion Planning of Energy Hub with Multiple Energy Infrastructures." IEEE Transactions on Smart Grid 6, no. 5 (September 2015): 2302-2311. DOI: 10.1109/TSG.2015.2390640.

Zlotnik, Anatoly, Line Roald, Scott Backhaus, Michael Chertkov, and Goran Andersson. "Coordinated Scheduling for Interdependent Electric Power and Natural Gas Infrastructures." IEEE Transactions on Power Systems 32, no. 1 (January 2017): 600-610. DOI: 10.1109/TPWRS.2016.2545522. 